\title{
QUASIPARTICLES IN CALCIUM DOPED YTTRIUM-IRON GARNETS
}

\author{
A. Lehmann-SzWeykowska ${ }^{a}$, R.J. WoJciechowski ${ }^{a}$, G.A. Gehring ${ }^{b}$ \\ AND I. TOBIJASZEWSKI ${ }^{a}$ \\ ${ }^{a}$ Institute of Physics, A. Mickiewicz University, 60-769 Poznań, Poland \\ ${ }^{b}$ Department of Physics, University of Sheffield S3 7RH, U.K.
}

In yttrium-iron garnets an electrical charge is carried by quasiparticles based on compensating holes which move either at oxygen or iron ions of the tetragonal sublattice. The quasiparticles can also get localized because of their coupling to optical phonons on the iron sites. A multi-band model of holes coupled to phonons is applied to find additional energy levels in the gap between the lower of the narrow $3 d$ bands and the wide $2 p$ band, whose occurrence plays a crucial role in a conductivity mechanism. The density of states is considerably modified by the interaction between the holes and local distortion of the lattice.

PACS numbers: $72.80 . \mathrm{Ga}, \mathbf{7 2 . 2 0 . J v}$

\section{Introduction}

Doping of magnetic garnets with non-trivalent ions increases their room temperature conductivity to nearly that of standard semiconductors [1]. The $\mathrm{Ca}^{2+}$-substituted yttrium-iron garnet (YIG) is an example of the charge-uncompensated doping. The calcium ions go into the dodecahedral $(c)$ sublattice. The thermopower measurements show that $\mathrm{Ca}^{2+}:$ YIG is a $p$-type semiconductor. Resistivity as low as $10^{2} \Omega \mathrm{cm}$ at $300 \mathrm{~K}$ can be achieved by this substitution while the original resistivity of pure YIG is higher than $10^{12} \Omega \mathrm{cm}$ at room temperatures. The activation energy is between 0.2 and $0.4 \mathrm{eV}$. An unusual increase in the conductivity by several orders of magnitude is observed in low temperatures in a partially compensated p-type CaGe:YIG, in garnet films when illuminated by white light and/or when placed in a high electric field [2]. However, the mechanism of the conduction process in the high conductivity state and its relationship to the normal state remains an open question. On the basis of measurements of the dc electrical conductivity and the Seebeck coefficient on single crystals, it was found that above room temperature both for $n$-type and $p$-type YIG, the large polaron band conduction is in the best agreement with the experiments [3]. 


\section{Model and results}

The main objective of this paper is to propose a possible conduction mechanism for the $p$-type YIG and discuss it specifically in the low-temperature region.

The model used throughout this paper is a three-dimensional periodic Anderson Hamiltonian. The first approximation is based on an analysis of possible occupation of different sites by compensating holes. Since the holes at tetragonal iron sites are more favoured than those at octahedral iron sites (by $4.2 \mathrm{eV}$ ) as well as at oxygen sites (by $1.6 \mathrm{eV}$ ) [4], the octahedral iron sublattice is completely eliminated. The subsequent approximation is even more drastic. In this short paper we will treat $d$ and $p$ electrons as narrow-band and wide-band $s$ electrons, respectively.

The Anderson Hamiltonian extended to include the phonons and their coupling to the electron system, takes the following form:

$$
H=H_{d}+H_{p}+H_{p-d}+H_{\mathrm{ph}}
$$

where

$$
\begin{aligned}
& H_{d}=\sum_{i, \sigma}\left[\varepsilon_{d}-\mu-\lambda_{1}\left(b_{i}^{\dagger}+b_{i}\right)\right] n_{i \sigma}^{d}+\frac{U}{2} \sum_{i \sigma} n_{i \sigma}^{d} n_{i-\sigma}^{d}+\sum_{i \neq j, \sigma} t_{i j}^{d} d_{i \sigma}^{\dagger} d_{j \sigma} \\
& H_{p}=(\varepsilon-\mu) \sum_{k, \sigma} n_{k \sigma}^{p}+\sum_{k \neq l, \sigma} t_{k l}^{p} c_{k \sigma}^{\dagger} c_{k \sigma}, \\
& H_{p-d}=\sum_{i \in d} \sum_{k \in p} \sum_{\sigma}\left\{V_{i k}\left[1+\lambda_{2}\left(b_{i}^{\dagger}+b_{i}\right)\right] d_{i \sigma}^{\dagger} c_{k \sigma}+h c\right\}
\end{aligned}
$$

As seen, the Hubbard Hamiltonian [5] for the narrow-band $(d)$ holes, is treated in the atomic limit. The hopping integral $t_{k l}$ in $H_{p}$ as well as the hybridization parameters $V_{i k}$ in $H_{p-d}$ are limited to nearest neighbours. The symbols $d_{i \sigma}$ and $c_{k \sigma}$ stand for the annihilation operators for a hole with spin $\sigma$ at the tetragonal iron site $(i)$ or the oxygen site $(k)$, respectively. The atomic energies of non-interacting holes $\left(E_{d}\right.$ and $\left.E_{p}\right)$ are measured with respect to the chemical potential $\mu . b_{i}$ is the annihilation operator for an optical phonon at the site $(i) . H_{\mathrm{ph}}$ is a free particle phonon Hamiltonian with $\omega_{0}$ being their frequency. The parameter $\lambda_{1}$ and $\lambda_{2}$ express the strength of the coupling. Our present objective is to calculate renormalized energies of quasiparticles based on the holes. The maximum number of the quasiparticle energies is equal to seven. The problem has been solved in an approximation which assumes: (i) a static mean-field approach, where all the statistical averages of the occupation numbers are preserved and considered as external parameters which control the doping; (ii) a substitution of all 24 tetragonal iron sublattices by one representative which results in a considerable reduction of branches in the energy spectrum, the same is applied to the 96 oxygen sites of the crystal unit cell.

The simplest band structure of YIG which accommodates for experimental facts, consists of a wide $2 p$ oxygen valence band, and at least two (assuming only the tetragonal iron sublattice) narrow $3 d$ bands of highly localized iron electrons.

In order to explain a possible mechanism of the electrical conductivity in YIG and its derivatives, we have looked for additional narrow energy bands in the 
gap between the $3 d$ and valence bands. In terms of holes, both in pure and doped YIG, the lower $3 d$ level is always occupied. If extra holes appear in the system, they are localized in the additional energy bands. In the positional space, it can be interpreted as a localization at either oxygen or iron ions. As already mentioned before, the light can release the holes from their traps so they get excited to the higher lying wide $2 p$ band and become mobile. In our approach, the formation of these additional energy bands is attributed to the coupling between the holes and optical phonons at the tetragonal iron sites. The bound states of the holes can be classified as polarons. In the present paper, the question whether they are small, or rather band polarons, remains open. The activation energy, defined as a difference between the lower edge of a possible conductivity band and the Fermi level, can be found and compared to the experimental data. In our case, the lower edge of a

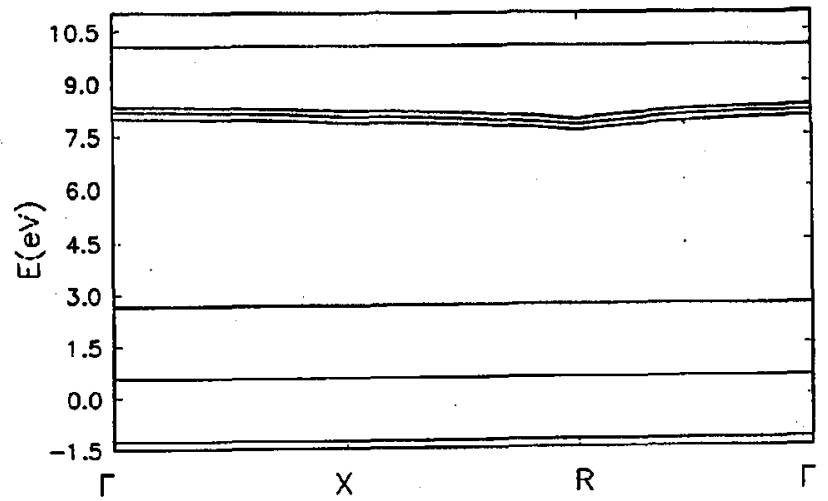

Fig. 1. Energy bands of the compensating holes interacting with optical phonons. Values of the system's parameters: $E_{d}=0, U=10 \mathrm{eV}, E_{p}=3.6 \mathrm{eV} V=1 \mathrm{eV}, \lambda_{1}=$ $2 \mathrm{eV}, \lambda_{2}=0.1 \mathrm{eV}, \omega_{0}=1 / 6 \mathrm{eV}$.

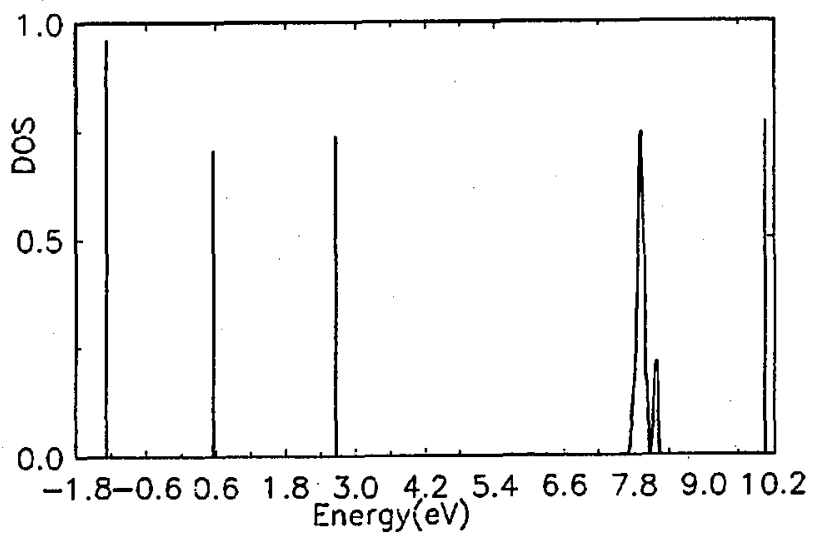

Fig. 2. Density of states vs. energy. All the parameters are the same as in Fig. 1. 
possible conductivity band is about $7.5 \mathrm{eV}$. The minimum value of the activation energy is equal to almost half of that in pure YIG, provided the doping places the Fermi level on the highest of the additional energy bands which is about $2.6 \mathrm{eV}$ in Fig. 2. An experimentally found activation energy can be used in estimating the strength of the hole-phonon coupling since the latter plays a key part in the determination of positions of the additional energy bands.

The numerical results are illustrated in Figs. 1 and 2. All seven branches of the energy spectrum display certain dispersion. The bands in Fig. 2 have a finite band width of about $.01 \mathrm{eV}$

This work is supported by the Committee for Scientific Research under grant No. 3 P408 00104.

\section{References}

[1] Yong Jin Song, Ph.D. Thesis, The Ohio State University, 1993.

[2] M. Parada vi-Horvath, S.H. Yuan, P.E. Wigen, P. DeGasperis, J. Magn. Magn. Mater. 119, 205 (1993).

[3] R. Metselaar, P.K. Larsen, in: Physics of Magnetic Garnets, Ed. Paoeletti, North-Holland, Amsterdam 1978, p. 417 and references therein.

[4] H. Donnerberg, C.R.A. Catlow, J. Phys., Condens. Matter 5, 2947 (1993).

[5] J. Hubbard, Proc. R. Soc. Lond. A 276, 238 (1963). 